\title{
An Overview of the Corporate Capital Structure in the Industries at the Tokyo Stock Exchange
}

\author{
Chikashi Tsuji ${ }^{1}$ \\ ${ }^{1}$ Faculty of Economics, Chuo University, Japan \\ Correspondence: Chikashi Tsuji, Faculty of Economics, Chuo University, 742-1 Higashinakano Hachioji-shi, \\ Tokyo 192-0393, Japan. Tel: 81-42-674-2211. E-mail: mail_sec_low@minos.ocn.ne.jp
}

Received: December 2, 2013

Accepted: December 21, 2013

Online Published: January 23, 2014

doi: $10.5539 /$ ibr.v7n2p100

URL: http://dx.doi.org/10.5539/ibr.v7n2p100

\begin{abstract}
This paper presents an overview of the differentials in the capital structures in various industries in Japan. More specifically, we first examine how capital structures of the industries at the Tokyo Stock Exchange (TSE) First Section are different. After that, we investigate whether financial risks associated with corporate debt ratios are rewarded with higher returns within industries. Main findings from our investigations are as follows. First, we find that the capital structure of each industry at the TSE First Section is statistically significantly much different. Second, we also find that financial risks associated with corporate debt ratios are not rewarded with future positive stock returns when we exclude the effects of industrial differentials of corporate leverage.
\end{abstract}

Keywords: capital structure, firm performance, industries at the Tokyo Stock Exchange, panel data analysis

\section{Introduction}

Capital structure is one of the traditional important research topics in corporate finance. Modigliani and Miller (1958) insisted that firms which have higher debt ratios are generally required higher stock returns. However, in the real world, corporate leverage shall be different in each industry. How is then the corporate capital structure different in each industry? Further, is the risk associated with the corporate debt ratio rewarded with higher return in the real world? We address two matters in this paper: first is the issue of the differentials of capital structures in various industries at the Tokyo Stock Exchange (TSE) First Section; second is the relationship between capital structure and stock return within each TSE industry.

With these viewpoints, the first objective of this paper is to empirically test the differentials of the capital structures in the industries at the TSE First Section. Our second objective is to test whether the financial risk connected with the corporate debt ratio is rewarded with higher stock return when we exclude the effects of industrial differentials of corporate leverage.

The contributions of this study are as follows. 1) First, we find that the capital structure of each industry at the TSE First Section is statistically significantly much different. 2) Second, we also reveal that the financial risk associated with the debt ratio is not rewarded with positive stock return when we exclude the effects of industrial differentials. The rest of the paper is organized as follows. Section 2 documents the literature review, section 3 describes our data and research design, Sections 4 to 6 explain our empirical results, and Section 7 summarizes the paper.

\section{Literature Review}

After Modigliani and Miller (1958), many studies focusing on capital structure have been implemented. Reviewing very recent studies, Margaritis and Psillaki (2010) explored the relations among capital structure, ownership structure, and firm performance. Kayo and Kimura (2011) investigated the influence of time-, firm-, industry- and country-level determinants of capital structure. Wang (2011) developed a theoretical model to examine the impacts of managerial entrenchment on capital structure and security valuation.

Further, Wu and Yeung (2012) found that firm growth type could parsimoniously predict significantly dispersed and persistently distinct future leverage ratios. Eisdorfer et al. (2013) examined how the similarity between the executive compensation leverage ratio and the corporate leverage ratio affected the quality of the corporate investment decisions. Feld et al. (2013) provided a quantitative review of the empirical literature on the tax impact on corporate debt financing. 
Moreover, Fier et al. (2013) exhibited the empirical evidence of a link between deviations from target leverage and internal capital markets activity. Schmid (2013) investigated the motives moving founders and their families to influence the capital structure decision.

In these recent studies reviewed above, Margaritis and Psillaki (2010), Kayo and Kimura (2011), Eisdorfer et al. (2013), Feld et al. (2013), and Schmid (2013) partially consider the issue of industry effects on capital structure. However, in these studies, the industrial differentials of capital structure are not the main focus of their investigations.

As understood from the above review of very recent literature, there is little empirical study that simultaneously focuses on two issues, namely, 1) the differentials of capital structures in various industries and 2) the relationship between capital structure and stock return within each industry. As stated, exploring these two issues is the objective of our study.

\section{Data and Research Design}

This section describes the data and methodology taken in this study. First, we exploit the data of the firms listed at the TSE First Section in Japan. All data are supplied by the Quick Corp. More specifically, we are interested in all firms listed at the TSE First Section; however, all characteristic information needed for our analyses is not obtained for all TSE First Section firms. Thus our full sample covers all firms whose data are enough available to implement our investigations. The sample period under our analyses is from the fiscal year of 1986 to 2012 .

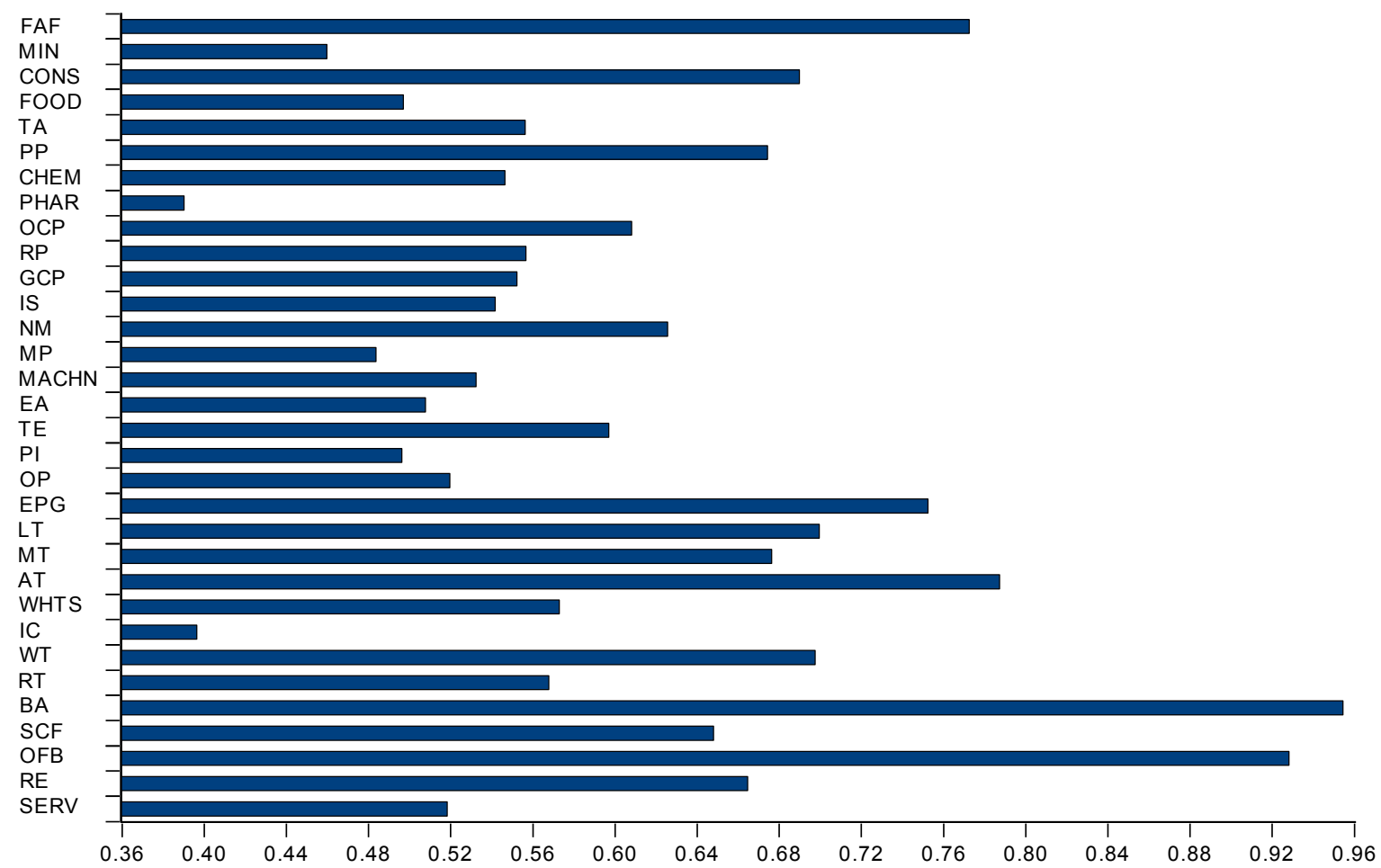

Figure 1. Differentials of the averages of the capital structures for the period from the fiscal year of 1986 to 2012: The case of the industries at the Tokyo Stock Exchange First Section

Notes: This figure shows the differentials of the average values of debt ratios of various industries at the Tokyo Stock Exchange First Section for the period from the fiscal year of 1986 to 2012. The debt ratios are measured by total book-value debts divided by total book-value assets. Industries at the TSE First Section are as follows; FAF: fishery, agriculture and forestry, MIN: mining, CONS: construction, FOOD: foods, TA: textiles and apparels, PP: pulp and paper, CHEM: chemicals, PHAR: pharmaceutical, OCP: oil and coal products, RP: rubber products, GCP: glass and ceramics products, IS: iron and steel, NM: nonferrous metals, MP: metal products, MACHN: machinery, EA: electric appliances, TE: transportation equipments, PI: precision instruments, OP: other products, EPG: electric power and gas, LT: land transportation, MT: marine transportation, AT: air transportation, WHTS: warehousing and harbor transportation services, IC: information and communication, WT: wholesale trade, RT: retail trade, BA: banks, SCF: securities and commodity futures, OFB: other financing business, RE: real estate, and SERV: services. 
Table 1. Capital structures of the industries at the Tokyo Stock Exchange First Section: The overview for the period for the fiscal year from 1986 to 2012

\begin{tabular}{|c|c|c|c|c|c|c|c|c|c|}
\hline \multicolumn{10}{|c|}{ Debt ratios of various industries at the TSE First Section } \\
\hline & 1986 & 1987 & 1988 & 1989 & 1990 & 1991 & 1992 & 1993 & 1994 \\
\hline FAF & 0.821 & 0.806 & 0.803 & 0.791 & 0.801 & 0.793 & 0.801 & 0.794 & 0.779 \\
\hline MIN & 0.511 & 0.485 & 0.473 & 0.485 & 0.474 & 0.421 & 0.430 & 0.424 & 0.438 \\
\hline CONS & 0.761 & 0.770 & 0.762 & 0.741 & 0.741 & 0.742 & 0.738 & 0.725 & 0.710 \\
\hline FOOD & 0.561 & 0.550 & 0.550 & 0.537 & 0.535 & 0.530 & 0.526 & 0.516 & 0.515 \\
\hline TA & 0.672 & 0.661 & 0.642 & 0.619 & 0.606 & 0.603 & 0.590 & 0.577 & 0.574 \\
\hline PP & 0.763 & 0.743 & 0.714 & 0.707 & 0.679 & 0.683 & 0.684 & 0.673 & 0.659 \\
\hline CHEM & 0.686 & 0.667 & 0.648 & 0.625 & 0.623 & 0.612 & 0.604 & 0.584 & 0.581 \\
\hline PHAR & 0.517 & 0.517 & 0.529 & 0.481 & 0.453 & 0.450 & 0.447 & 0.439 & 0.443 \\
\hline $\mathrm{OCP}$ & 0.651 & 0.649 & 0.670 & 0.639 & 0.650 & 0.613 & 0.598 & 0.579 & 0.570 \\
\hline $\mathrm{RP}$ & 0.717 & 0.710 & 0.695 & 0.673 & 0.661 & 0.652 & 0.626 & 0.596 & 0.566 \\
\hline GCP & 0.630 & 0.629 & 0.598 & 0.586 & 0.584 & 0.578 & 0.577 & 0.578 & 0.573 \\
\hline IS & 0.715 & 0.680 & 0.664 & 0.614 & 0.590 & 0.573 & 0.561 & 0.554 & 0.553 \\
\hline NM & 0.736 & 0.728 & 0.697 & 0.674 & 0.677 & 0.668 & 0.667 & 0.650 & 0.653 \\
\hline MP & 0.600 & 0.589 & 0.587 & 0.563 & 0.556 & 0.547 & 0.528 & 0.505 & 0.501 \\
\hline MACHN & 0.584 & 0.580 & 0.574 & 0.567 & 0.567 & 0.557 & 0.549 & 0.545 & 0.548 \\
\hline EA & 0.558 & 0.553 & 0.555 & 0.547 & 0.542 & 0.532 & 0.524 & 0.520 & 0.521 \\
\hline TE & 0.659 & 0.656 & 0.650 & 0.643 & 0.651 & 0.653 & 0.648 & 0.626 & 0.614 \\
\hline PI & 0.486 & 0.504 & 0.496 & 0.527 & 0.531 & 0.520 & 0.530 & 0.509 & 0.504 \\
\hline OP & 0.583 & 0.595 & 0.584 & 0.547 & 0.559 & 0.563 & 0.549 & 0.536 & 0.524 \\
\hline EPG & 0.751 & 0.742 & 0.743 & 0.749 & 0.755 & 0.764 & 0.773 & 0.779 & 0.786 \\
\hline LT & 0.782 & 0.764 & 0.738 & 0.718 & 0.721 & 0.723 & 0.716 & 0.722 & 0.723 \\
\hline MT & 0.825 & 0.826 & 0.803 & 0.779 & 0.785 & 0.758 & 0.754 & 0.751 & 0.737 \\
\hline AT & 0.667 & 0.724 & 0.732 & 0.764 & 0.780 & 0.799 & 0.818 & 0.823 & 0.796 \\
\hline WHTS & 0.657 & 0.672 & 0.645 & 0.615 & 0.619 & 0.618 & 0.610 & 0.601 & 0.603 \\
\hline IC & 0.497 & 0.513 & 0.497 & 0.457 & 0.427 & 0.406 & 0.426 & 0.411 & 0.416 \\
\hline WT & 0.779 & 0.778 & 0.773 & 0.759 & 0.754 & 0.745 & 0.735 & 0.719 & 0.719 \\
\hline RT & 0.607 & 0.578 & 0.565 & 0.543 & 0.542 & 0.575 & 0.561 & 0.562 & 0.558 \\
\hline BA & 0.967 & 0.967 & 0.965 & 0.962 & 0.962 & 0.962 & 0.959 & 0.959 & 0.958 \\
\hline $\mathrm{SCF}$ & 0.842 & 0.836 & 0.828 & 0.825 & 0.791 & 0.741 & 0.715 & 0.738 & 0.741 \\
\hline OFB & 0.941 & 0.939 & 0.938 & 0.933 & 0.935 & 0.935 & 0.933 & 0.930 & 0.928 \\
\hline RE & 0.678 & 0.696 & 0.693 & 0.669 & 0.675 & 0.696 & 0.689 & 0.681 & 0.671 \\
\hline SERV & 0.580 & 0.562 & 0.521 & 0.527 & 0.533 & 0.525 & 0.534 & 0.521 & 0.505 \\
\hline Average & 0.681 & 0.677 & 0.667 & 0.652 & 0.649 & 0.642 & 0.638 & 0.629 & 0.624 \\
\hline Max. & 0.967 & 0.967 & 0.965 & 0.962 & 0.962 & 0.962 & 0.959 & 0.959 & 0.958 \\
\hline Min. & 0.486 & 0.485 & 0.473 & 0.457 & 0.427 & 0.406 & 0.426 & 0.411 & 0.416 \\
\hline Std. Dev. & 0.120 & 0.120 & 0.121 & 0.124 & 0.127 & 0.130 & 0.130 & 0.134 & 0.132 \\
\hline
\end{tabular}




\begin{tabular}{|c|c|c|c|c|c|c|c|c|c|}
\hline \multicolumn{10}{|c|}{ Debt ratios of various industries at the TSE First Section } \\
\hline & 1995 & 1996 & 1997 & 1998 & 1999 & 2000 & 2001 & 2002 & 2003 \\
\hline FAF & 0.777 & 0.774 & 0.756 & 0.750 & 0.730 & 0.731 & 0.774 & 0.773 & 0.744 \\
\hline MIN & 0.453 & 0.471 & 0.463 & 0.460 & 0.489 & 0.486 & 0.522 & 0.572 & 0.541 \\
\hline CONS & 0.713 & 0.704 & 0.695 & 0.695 & 0.685 & 0.699 & 0.692 & 0.698 & 0.678 \\
\hline FOOD & 0.512 & 0.500 & 0.509 & 0.503 & 0.495 & 0.505 & 0.503 & 0.483 & 0.470 \\
\hline TA & 0.569 & 0.560 & 0.562 & 0.563 & 0.563 & 0.573 & 0.557 & 0.542 & 0.520 \\
\hline PP & 0.653 & 0.677 & 0.680 & 0.686 & 0.689 & 0.669 & 0.669 & 0.670 & 0.653 \\
\hline CHEM & 0.574 & 0.562 & 0.553 & 0.544 & 0.537 & 0.532 & 0.520 & 0.510 & 0.493 \\
\hline PHAR & 0.449 & 0.440 & 0.418 & 0.421 & 0.397 & 0.406 & 0.377 & 0.366 & 0.339 \\
\hline OCP & 0.574 & 0.588 & 0.583 & 0.580 & 0.608 & 0.595 & 0.583 & 0.597 & 0.583 \\
\hline RP & 0.544 & 0.528 & 0.515 & 0.521 & 0.519 & 0.515 & 0.526 & 0.518 & 0.515 \\
\hline GCP & 0.570 & 0.573 & 0.554 & 0.564 & 0.570 & 0.559 & 0.545 & 0.542 & 0.527 \\
\hline IS & 0.550 & 0.536 & 0.529 & 0.533 & 0.554 & 0.559 & 0.556 & 0.552 & 0.532 \\
\hline NM & 0.648 & 0.643 & 0.638 & 0.636 & 0.621 & 0.604 & 0.593 & 0.586 & 0.580 \\
\hline MP & 0.493 & 0.492 & 0.481 & 0.471 & 0.484 & 0.491 & 0.462 & 0.454 & 0.448 \\
\hline MACHN & 0.555 & 0.555 & 0.552 & 0.530 & 0.527 & 0.543 & 0.543 & 0.545 & 0.524 \\
\hline EA & 0.522 & 0.509 & 0.502 & 0.506 & 0.503 & 0.516 & 0.505 & 0.509 & 0.495 \\
\hline TE & 0.598 & 0.590 & 0.577 & 0.587 & 0.590 & 0.586 & 0.585 & 0.585 & 0.570 \\
\hline PI & 0.516 & 0.496 & 0.476 & 0.460 & 0.490 & 0.515 & 0.484 & 0.499 & 0.497 \\
\hline $\mathrm{OP}$ & 0.524 & 0.524 & 0.506 & 0.499 & 0.511 & 0.527 & 0.526 & 0.523 & 0.520 \\
\hline EPG & 0.793 & 0.798 & 0.798 & 0.799 & 0.784 & 0.764 & 0.759 & 0.752 & 0.736 \\
\hline LT & 0.725 & 0.723 & 0.712 & 0.711 & 0.711 & 0.719 & 0.706 & 0.706 & 0.693 \\
\hline MT & 0.736 & 0.725 & 0.742 & 0.735 & 0.758 & 0.732 & 0.703 & 0.702 & 0.648 \\
\hline AT & 0.790 & 0.785 & 0.794 & 0.948 & 0.776 & 0.771 & 0.785 & 0.813 & 0.854 \\
\hline WHTS & 0.593 & 0.584 & 0.569 & 0.566 & 0.575 & 0.569 & 0.563 & 0.564 & 0.545 \\
\hline IC & 0.408 & 0.432 & 0.418 & 0.396 & 0.352 & 0.359 & 0.379 & 0.391 & 0.374 \\
\hline WT & 0.724 & 0.719 & 0.711 & 0.703 & 0.700 & 0.703 & 0.690 & 0.692 & 0.676 \\
\hline RT & 0.555 & 0.548 & 0.555 & 0.566 & 0.566 & 0.585 & 0.626 & 0.617 & 0.606 \\
\hline BA & 0.959 & 0.958 & 0.960 & 0.955 & 0.954 & 0.946 & 0.951 & 0.953 & 0.950 \\
\hline $\mathrm{SCF}$ & 0.787 & 0.788 & 0.786 & 0.809 & 0.758 & 0.685 & 0.485 & 0.512 & 0.467 \\
\hline OFB & 0.930 & 0.930 & 0.933 & 0.931 & 0.927 & 0.947 & 0.947 & 0.943 & 0.933 \\
\hline RE & 0.673 & 0.679 & 0.695 & 0.691 & 0.696 & 0.700 & 0.723 & 0.675 & 0.651 \\
\hline SERV & 0.507 & 0.514 & 0.495 & 0.485 & 0.485 & 0.504 & 0.529 & 0.536 & 0.539 \\
\hline Average & 0.624 & 0.622 & 0.616 & 0.619 & 0.613 & 0.612 & 0.605 & 0.606 & 0.591 \\
\hline Max. & 0.959 & 0.958 & 0.960 & 0.955 & 0.954 & 0.947 & 0.951 & 0.953 & 0.950 \\
\hline Min. & 0.408 & 0.432 & 0.418 & 0.396 & 0.352 & 0.359 & 0.377 & 0.366 & 0.339 \\
\hline Std. Dev. & 0.134 & 0.134 & 0.139 & 0.149 & 0.138 & 0.132 & 0.136 & 0.136 & 0.139 \\
\hline
\end{tabular}




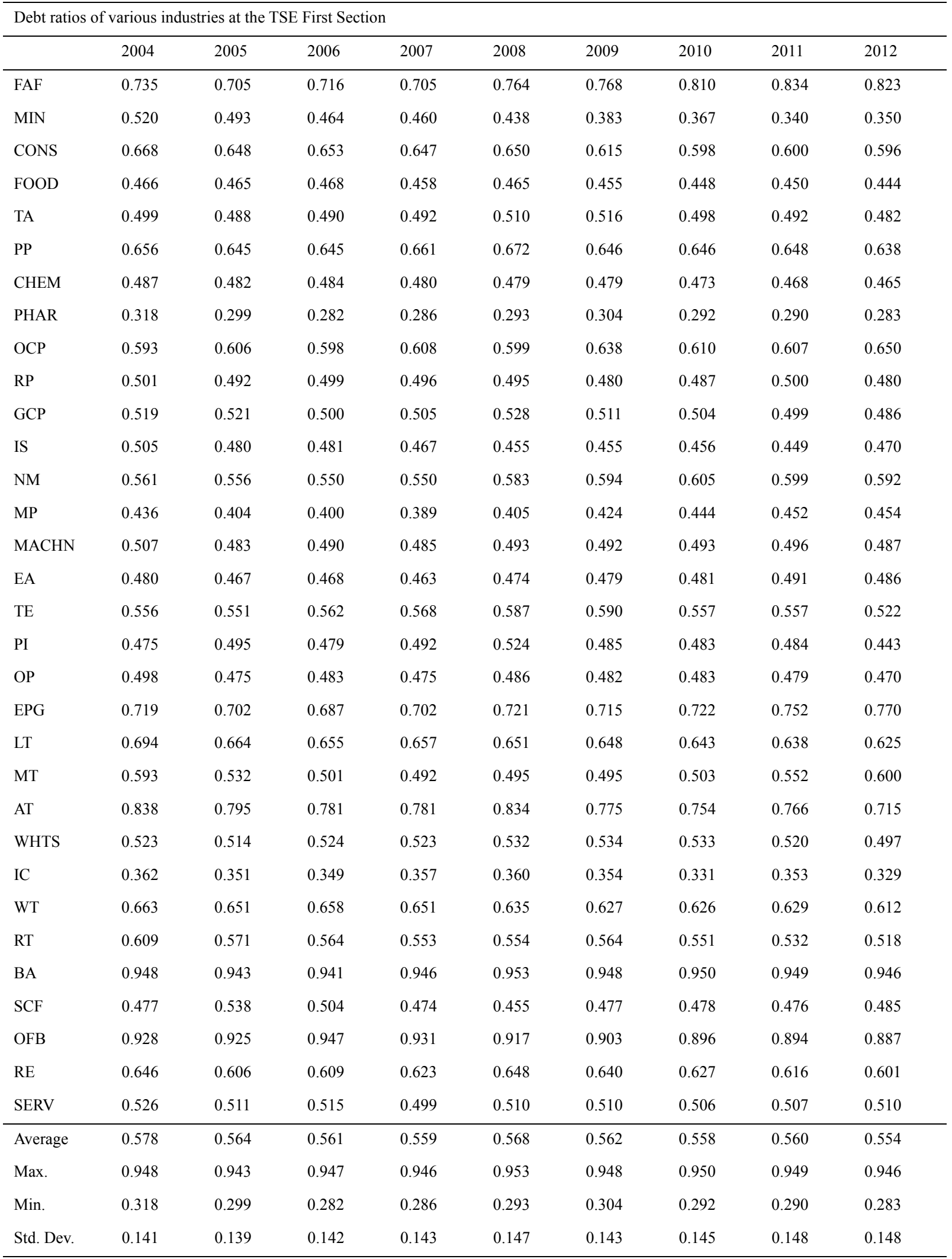

Notes: This table exhibits the average values of the debt ratios of various industries at the Tokyo Stock Exchange First Section. The values of the capital structure in the table are measured by total book-value debts divided by total book-value assets. 'Std. Dev.' denotes the standard deviation. Further, 'Max.' and 'Min.' denote the maximum and minimum values, respectively. 
Table 2. Results of the Welch's tests regarding the statistical differential of capital structure of each industry at the Tokyo Stock Exchange First Section: Evidence for the period from the fiscal year of 1986 to 2012

\begin{tabular}{|c|c|c|c|c|c|c|c|c|c|}
\hline \multicolumn{10}{|c|}{ Results of the Welch's tests for the capital structure differentials } \\
\hline & \multicolumn{9}{|c|}{$p$-values of the Welch's tests } \\
\hline & \multicolumn{9}{|c|}{ Fiscal year } \\
\hline & 1986 & 1987 & 1988 & 1989 & 1990 & 1991 & 1992 & 1993 & 1994 \\
\hline FAF & 0.139 & 0.165 & 0.159 & 0.144 & 0.106 & $0.095^{+}$ & 0.119 & 0.105 & 0.101 \\
\hline MIN & 0.116 & 0.122 & 0.118 & 0.170 & 0.185 & 0.173 & 0.198 & 0.207 & 0.231 \\
\hline CONS & $0.000^{+++}$ & $0.000^{+++}$ & $0.000^{+++}$ & $0.000^{+++}$ & $0.000^{+++}$ & $0.000^{+++}$ & $0.000^{+++}$ & $0.000^{+++}$ & $0.000^{+++}$ \\
\hline FOOD & $0.000^{---}$ & $0.000^{---}$ & $0.000^{---}$ & $0.000^{---}$ & $0.000^{---}$ & $0.000^{---}$ & $0.001^{---}$ & $0.001^{---}$ & $0.002^{---}$ \\
\hline $\mathrm{TA}$ & 0.331 & 0.373 & 0.482 & 0.449 & 0.343 & 0.362 & 0.307 & 0.300 & 0.305 \\
\hline PP & $0.004^{+++}$ & $0.024^{++}$ & $0.058^{+}$ & $0.066^{+}$ & $0.027^{++}$ & $0.032^{++}$ & $0.041^{++}$ & $0.026^{++}$ & $0.052^{+}$ \\
\hline CHEM & $0.083^{+}$ & 0.198 & 0.345 & 0.465 & 0.438 & 0.419 & 0.406 & 0.243 & 0.256 \\
\hline PHAR & $0.000^{---}$ & $0.000^{---}$ & $0.000^{---}$ & $0.000^{---}$ & $0.000^{---}$ & $0.000^{---}$ & $0.000^{---}$ & $0.000^{---}$ & $0.000^{---}$ \\
\hline $\mathrm{OCP}$ & 0.485 & 0.497 & 0.409 & 0.458 & 0.420 & 0.493 & 0.468 & 0.442 & 0.422 \\
\hline $\mathrm{RP}$ & 0.198 & 0.175 & 0.130 & 0.144 & 0.195 & 0.167 & 0.349 & 0.475 & 0.264 \\
\hline GCP & 0.213 & 0.239 & $0.097^{-}$ & 0.123 & 0.131 & 0.141 & 0.168 & 0.272 & 0.267 \\
\hline IS & $0.042^{++}$ & 0.203 & 0.215 & 0.379 & 0.163 & $0.082^{-}$ & $0.077^{-}$ & 0.116 & 0.126 \\
\hline NM & $0.019^{++}$ & $0.024^{++}$ & $0.075^{+}$ & $0.066^{+}$ & $0.044^{++}$ & $0.069^{+}$ & $0.055^{+}$ & $0.085^{+}$ & $0.058^{+}$ \\
\hline MP & $0.048^{--}$ & $0.029^{--}$ & $0.040^{--}$ & $0.012^{--}$ & $0.009^{---}$ & $0.007^{---}$ & $0.002^{---}$ & $0.001^{---}$ & $0.001^{---}$ \\
\hline MACHN & $0.001^{---}$ & $0.001^{---}$ & $0.001^{---}$ & $0.004^{---}$ & $0.006^{---}$ & $0.006^{---}$ & $0.008^{---}$ & $0.022^{--}$ & $0.045^{--}$ \\
\hline EA & $0.000^{---}$ & $0.000^{---}$ & $0.000^{---}$ & $0.000^{---}$ & $0.000^{---}$ & $0.000^{---}$ & $0.000^{---}$ & $0.000^{---}$ & $0.001^{---}$ \\
\hline $\mathrm{TE}$ & 0.462 & 0.410 & 0.330 & 0.209 & 0.101 & $0.064^{+}$ & $0.063^{+}$ & 0.158 & 0.243 \\
\hline PI & $0.001^{---}$ & $0.005^{---}$ & $0.007^{---}$ & $0.022^{--}$ & $0.026^{--}$ & $0.022^{--}$ & $0.046^{--}$ & $0.053^{-}$ & $0.051^{-}$ \\
\hline OP & $0.026^{--}$ & $0.073^{-}$ & $0.071^{-}$ & $0.006^{---}$ & $0.011^{--}$ & $0.023^{--}$ & $0.021^{--}$ & $0.023^{--}$ & $0.016^{--}$ \\
\hline EPG & $0.002^{+++}$ & $0.004^{+++}$ & $0.001^{+++}$ & $0.000^{+++}$ & $0.000^{+++}$ & $0.000^{+++}$ & $0.000^{+++}$ & $0.000^{+++}$ & $0.000^{+++}$ \\
\hline LT & $0.000^{+++}$ & $0.000^{+++}$ & $0.001^{+++}$ & $0.002^{+++}$ & $0.002^{+++}$ & $0.002^{+++}$ & $0.003^{+++}$ & $0.000^{+++}$ & $0.000^{+++}$ \\
\hline MT & $0.000^{+++}$ & $0.000^{+++}$ & $0.000^{+++}$ & $0.002^{+++}$ & $0.000^{+++}$ & $0.000^{+++}$ & $0.000^{+++}$ & $0.000^{+++}$ & $0.000^{+++}$ \\
\hline $\mathrm{AT}$ & 0.372 & 0.126 & $0.075^{+}$ & $0.063^{+}$ & $0.069^{+}$ & $0.056^{+}$ & $0.045^{++}$ & $0.037^{++}$ & $0.072^{+}$ \\
\hline WHTS & 0.497 & 0.308 & 0.453 & 0.382 & 0.487 & 0.467 & 0.485 & 0.471 & 0.414 \\
\hline IC & $0.043^{--}$ & $0.048^{--}$ & $0.030^{--}$ & $0.040^{--}$ & $0.034^{--}$ & $0.030^{--}$ & $0.028^{--}$ & $0.035^{--}$ & $0.037^{--}$ \\
\hline WT & $0.000^{+++}$ & $0.000^{+++}$ & $0.000^{+++}$ & $0.000^{+++}$ & $0.000^{+++}$ & $0.000^{+++}$ & $0.000^{+++}$ & $0.000^{+++}$ & $0.000^{+++}$ \\
\hline RT & $0.048^{--}$ & $0.012^{--}$ & $0.005^{---}$ & $0.005^{---}$ & $0.005^{---}$ & $0.067^{-}$ & $0.048^{--}$ & $0.093^{-}$ & 0.119 \\
\hline BA & $0.000^{+++}$ & $0.000^{+++}$ & $0.000^{+++}$ & $0.000^{+++}$ & $0.000^{+++}$ & $0.000^{+++}$ & $0.000^{+++}$ & $0.000^{+++}$ & $0.000^{+++}$ \\
\hline SCF & $0.000^{+++}$ & $0.000^{+++}$ & $0.002^{+++}$ & $0.003^{+++}$ & $0.004^{+++}$ & $0.018^{++}$ & $0.022^{+++}$ & $0.002^{+++}$ & $0.000^{+++}$ \\
\hline OFB & $0.000^{+++}$ & $0.000^{+++}$ & $0.000^{+++}$ & $0.000^{+++}$ & $0.001^{+++}$ & $0.000^{+++}$ & $0.001^{+++}$ & $0.001^{+++}$ & $0.001^{+++}$ \\
\hline $\mathrm{RE}$ & 0.299 & 0.149 & 0.115 & 0.169 & 0.147 & $0.050^{++}$ & $0.067^{+}$ & $0.073^{+}$ & 0.104 \\
\hline SERV & $0.072^{-}$ & $0.089^{-}$ & $0.043^{--}$ & $0.077^{-}$ & 0.101 & 0.117 & 0.169 & 0.171 & 0.139 \\
\hline
\end{tabular}


Results of the Welch's tests for the capital structure differentials

\begin{tabular}{|c|c|c|c|c|c|c|c|c|c|}
\hline & \multicolumn{9}{|c|}{$p$-values of the Welch's tests } \\
\hline & \multicolumn{9}{|c|}{ Fiscal year } \\
\hline & 1995 & 1996 & 1997 & 1998 & 1999 & 2000 & 2001 & 2002 & 2003 \\
\hline FAF & 0.104 & 0.121 & 0.148 & 0.134 & 0.155 & 0.145 & $0.033^{++}$ & $0.002^{+++}$ & $0.003^{+++}$ \\
\hline MIN & 0.256 & 0.302 & 0.305 & 0.297 & 0.353 & 0.334 & 0.415 & 0.498 & 0.473 \\
\hline CONS & $0.000^{+++}$ & $0.000^{+++}$ & $0.000^{+++}$ & $0.000^{+++}$ & $0.000^{+++}$ & $0.000^{+++}$ & $0.000^{+++}$ & $0.000^{+++}$ & $0.000^{+++}$ \\
\hline FOOD & $0.002^{---}$ & $0.001^{---}$ & $0.005^{---}$ & $0.005^{---}$ & $0.004^{---}$ & $0.005^{---}$ & $0.010^{---}$ & $0.004^{---}$ & $0.002^{---}$ \\
\hline TA & 0.296 & 0.265 & 0.333 & 0.359 & 0.377 & 0.410 & 0.326 & 0.240 & 0.170 \\
\hline PP & $0.074^{+}$ & $0.032^{++}$ & $0.027^{++}$ & $0.019^{++}$ & $0.005^{+++}$ & $0.030^{++}$ & $0.023^{++}$ & $0.027^{++}$ & $0.018^{++}$ \\
\hline CHEM & 0.174 & 0.108 & $0.093^{-}$ & $0.067^{-}$ & $0.033^{--}$ & $0.008^{---}$ & $0.006^{---}$ & $0.002^{---}$ & $0.001^{---}$ \\
\hline PHAR & $0.000^{---}$ & $0.000^{---}$ & $0.000^{---}$ & $0.000^{---}$ & $0.000^{---}$ & $0.000^{---}$ & $0.000^{---}$ & $0.000^{---}$ & $0.000^{---}$ \\
\hline $\mathrm{OCP}$ & 0.441 & 0.500 & 0.495 & 0.493 & 0.402 & 0.457 & 0.480 & 0.430 & 0.427 \\
\hline RP & 0.161 & 0.113 & 0.107 & 0.140 & 0.150 & $0.083^{-}$ & 0.141 & 0.104 & 0.136 \\
\hline GCP & 0.240 & 0.331 & 0.216 & 0.339 & 0.429 & 0.247 & 0.173 & 0.173 & 0.167 \\
\hline IS & 0.112 & $0.080^{-}$ & $0.085^{-}$ & 0.138 & 0.310 & 0.284 & 0.312 & 0.308 & 0.257 \\
\hline NM & $0.054^{+}$ & $0.057^{+}$ & $0.055^{+}$ & $0.056^{+}$ & 0.118 & 0.282 & 0.345 & 0.394 & 0.324 \\
\hline MP & $0.000^{---}$ & $0.000^{---}$ & $0.001^{---}$ & $0.000^{---}$ & $0.002^{---}$ & $0.001^{---}$ & $0.003^{---}$ & $0.002^{---}$ & $0.003^{---}$ \\
\hline MACHN & $0.080^{-}$ & 0.113 & 0.140 & $0.045^{--}$ & $0.031^{--}$ & $0.061^{-}$ & $0.099^{-}$ & 0.141 & $0.087^{-}$ \\
\hline EA & $0.001^{---}$ & $0.000^{---}$ & $0.000^{---}$ & $0.002^{---}$ & $0.001^{---}$ & $0.003^{---}$ & $0.003^{---}$ & $0.006^{---}$ & $0.005^{---}$ \\
\hline TE & 0.448 & 0.465 & 0.450 & 0.378 & 0.321 & 0.444 & 0.382 & 0.360 & 0.335 \\
\hline PI & $0.074^{-}$ & $0.052^{-}$ & $0.031^{--}$ & $0.029^{--}$ & $0.050^{--}$ & 0.116 & $0.071^{-}$ & 0.123 & 0.151 \\
\hline OP & $0.012^{--}$ & $0.016^{--}$ & $0.007^{---}$ & $0.008^{---}$ & $0.033^{--}$ & $0.062^{-}$ & $0.094^{-}$ & 0.101 & 0.191 \\
\hline EPG & $0.000^{+++}$ & $0.000^{+++}$ & $0.000^{+++}$ & $0.000^{+++}$ & $0.000^{+++}$ & $0.000^{+++}$ & $0.000^{+++}$ & $0.000^{+++}$ & $0.000^{+++}$ \\
\hline LT & $0.000^{+++}$ & $0.000^{+++}$ & $0.000^{+++}$ & $0.000^{+++}$ & $0.000^{+++}$ & $0.000^{+++}$ & $0.000^{+++}$ & $0.001^{+++}$ & $0.002^{+++}$ \\
\hline MT & $0.000^{+++}$ & $0.001^{+++}$ & $0.000^{+++}$ & $0.000^{+++}$ & $0.000^{+++}$ & $0.000^{+++}$ & $0.000^{+++}$ & $0.000^{+++}$ & $0.008^{+++}$ \\
\hline AT & $0.075^{+}$ & $0.060^{+}$ & $0.069^{+}$ & $0.087^{+}$ & 0.126 & 0.127 & 0.123 & $0.092^{+}$ & $0.032^{++}$ \\
\hline WHTS & 0.493 & 0.468 & 0.404 & 0.407 & 0.490 & 0.417 & 0.418 & 0.438 & 0.415 \\
\hline IC & $0.035^{--}$ & $0.057^{-}$ & $0.037^{--}$ & $0.027^{--}$ & $0.000^{---}$ & $0.003^{---}$ & $0.013^{--}$ & $0.008^{---}$ & $0.004^{---}$ \\
\hline WT & $0.000^{+++}$ & $0.000^{+++}$ & $0.000^{+++}$ & $0.000^{+++}$ & $0.000^{+++}$ & $0.000^{+++}$ & $0.000^{+++}$ & $0.000^{+++}$ & $0.000^{+++}$ \\
\hline RT & 0.109 & 0.107 & 0.215 & 0.365 & 0.389 & 0.469 & 0.112 & 0.127 & $0.100^{+}$ \\
\hline $\mathrm{BA}$ & $0.000^{+++}$ & $0.000^{+++}$ & $0.000^{+++}$ & $0.000^{+++}$ & $0.000^{+++}$ & $0.000^{+++}$ & $0.000^{+++}$ & $0.000^{+++}$ & $0.000^{+++}$ \\
\hline $\mathrm{SCF}$ & $0.000^{+++}$ & $0.003^{+++}$ & $0.008^{+++}$ & $0.002^{+++}$ & $0.031^{++}$ & 0.127 & 0.119 & 0.137 & 0.180 \\
\hline OFB & $0.000^{+++}$ & $0.000^{+++}$ & $0.000^{+++}$ & $0.000^{+++}$ & $0.000^{+++}$ & $0.000^{+++}$ & $0.000^{+++}$ & $0.000^{+++}$ & $0.000^{+++}$ \\
\hline $\mathrm{RE}$ & 0.101 & $0.068^{+}$ & $0.038^{++}$ & $0.042^{++}$ & $0.038^{++}$ & $0.039^{++}$ & $0.075^{+}$ & $0.066^{+}$ & $0.089^{+}$ \\
\hline SERV & 0.141 & 0.162 & 0.158 & 0.153 & 0.163 & 0.205 & 0.311 & 0.348 & 0.414 \\
\hline
\end{tabular}


Results of the Welch's tests for the capital structure differentials

\begin{tabular}{|c|c|c|c|c|c|c|c|c|c|}
\hline & $p$-values 0 & ne Welch's & & & & & & & \\
\hline & Fiscal yea & & & & & & & & \\
\hline & 2004 & 2005 & 2006 & 2007 & 2008 & 2009 & 2010 & 2011 & 2012 \\
\hline FAF & $0.003^{+++}$ & $0.004^{+++}$ & $0.000^{+++}$ & $0.000^{+++}$ & $0.047^{++}$ & $0.052^{+}$ & $0.032^{++}$ & $0.030^{++}$ & $0.035^{++}$ \\
\hline MIN & 0.459 & 0.430 & 0.352 & 0.355 & 0.305 & 0.173 & 0.147 & 0.104 & 0.124 \\
\hline CONS & $0.000^{+++}$ & $0.000^{+++}$ & $0.000^{+++}$ & $0.000^{+++}$ & $0.000^{+++}$ & $0.000^{+++}$ & $0.001^{+++}$ & $0.001^{+++}$ & $0.000^{+++}$ \\
\hline FOOD & $0.004^{---}$ & $0.010^{---}$ & $0.017^{--}$ & $0.010^{---}$ & $0.012^{--}$ & $0.005^{---}$ & $0.003^{---}$ & $0.004^{---}$ & $0.005^{---}$ \\
\hline $\mathrm{TA}$ & 0.124 & 0.150 & 0.158 & 0.202 & 0.294 & 0.384 & 0.272 & 0.218 & 0.192 \\
\hline PP & $0.011^{++}$ & $0.007^{+++}$ & $0.018^{++}$ & $0.007^{+++}$ & $0.002^{+++}$ & $0.004^{+++}$ & $0.005^{+++}$ & $0.010^{+++}$ & $0.011^{++}$ \\
\hline CHEM & $0.002^{---}$ & $0.006^{---}$ & $0.008^{---}$ & $0.008^{---}$ & $0.006^{---}$ & $0.008^{---}$ & $0.008^{---}$ & $0.004^{---}$ & $0.008^{---}$ \\
\hline PHAR & $0.000^{---}$ & $0.000^{---}$ & $0.000^{---}$ & $0.000^{---}$ & $0.000^{---}$ & $0.000^{---}$ & $0.000^{---}$ & $0.000^{---}$ & $0.000^{---}$ \\
\hline OCP & 0.355 & 0.274 & 0.288 & 0.237 & 0.268 & 0.166 & 0.231 & 0.235 & 0.166 \\
\hline $\mathrm{RP}$ & 0.126 & 0.167 & 0.231 & 0.255 & 0.240 & 0.144 & 0.223 & 0.311 & 0.227 \\
\hline GCP & 0.196 & 0.384 & 0.155 & 0.259 & 0.440 & 0.319 & 0.294 & 0.240 & 0.200 \\
\hline IS & 0.137 & $0.081^{-}$ & $0.082^{-}$ & $0.057^{-}$ & $0.032^{--}$ & $0.041^{--}$ & $0.054^{-}$ & $0.044^{--}$ & 0.218 \\
\hline NM & 0.375 & 0.296 & 0.329 & 0.292 & 0.162 & $0.092^{+}$ & $0.047^{++}$ & $0.048^{++}$ & $0.037^{++}$ \\
\hline MP & $0.003^{---}$ & $0.001^{---}$ & $0.001^{---}$ & $0.002^{---}$ & $0.008^{---}$ & $0.009^{---}$ & $0.042^{--}$ & $0.043^{--}$ & $0.087^{-}$ \\
\hline MACHN & $0.049^{--}$ & $0.018^{--}$ & $0.031^{--}$ & $0.028^{--}$ & $0.056^{-}$ & $0.073^{-}$ & $0.099^{-}$ & 0.108 & 0.108 \\
\hline EA & $0.004^{---}$ & $0.004^{---}$ & $0.004^{---}$ & $0.003^{---}$ & $0.012^{--}$ & $0.023^{--}$ & $0.041^{--}$ & $0.082^{-}$ & 0.103 \\
\hline $\mathrm{TE}$ & 0.345 & 0.203 & $0.082^{+}$ & $0.047^{++}$ & $0.029^{++}$ & $0.015^{++}$ & 0.110 & 0.106 & 0.425 \\
\hline PI & 0.110 & 0.252 & 0.150 & 0.288 & 0.448 & 0.217 & 0.248 & 0.255 & $0.099^{-}$ \\
\hline OP & 0.123 & $0.059^{-}$ & $0.063^{-}$ & $0.042^{--}$ & $0.082^{-}$ & $0.081^{-}$ & 0.112 & $0.095^{-}$ & $0.082^{-}$ \\
\hline EPG & $0.000^{+++}$ & $0.000^{+++}$ & $0.000^{+++}$ & $0.000^{+++}$ & $0.000^{+++}$ & $0.000^{+++}$ & $0.000^{+++}$ & $0.000^{+++}$ & $0.000^{+++}$ \\
\hline LT & $0.001^{+++}$ & $0.003^{+++}$ & $0.004^{+++}$ & $0.003^{+++}$ & $0.007^{+++}$ & $0.006^{+++}$ & $0.005^{+++}$ & $0.007^{+++}$ & $0.009^{+++}$ \\
\hline MT & $0.082^{+}$ & 0.476 & 0.279 & 0.262 & 0.275 & 0.330 & 0.396 & 0.367 & 0.175 \\
\hline AT & $0.001^{+++}$ & $0.021^{++}$ & $0.046^{++}$ & $0.039^{++}$ & $0.000^{+++}$ & $0.031^{++}$ & $0.024^{++}$ & $0.044^{++}$ & 0.108 \\
\hline WHTS & 0.353 & 0.389 & 0.454 & 0.477 & 0.494 & 0.463 & 0.436 & 0.470 & 0.376 \\
\hline IC & $0.009^{---}$ & $0.015^{--}$ & $0.014^{--}$ & $0.030^{--}$ & $0.010^{---}$ & $0.007^{---}$ & $0.001^{---}$ & $0.014^{--}$ & $0.021^{--}$ \\
\hline WT & $0.000^{+++}$ & $0.000^{+++}$ & $0.000^{+++}$ & $0.000^{+++}$ & $0.000^{+++}$ & $0.000^{+++}$ & $0.000^{+++}$ & $0.000^{+++}$ & $0.000^{+++}$ \\
\hline RT & $0.076^{+}$ & 0.132 & 0.166 & 0.215 & 0.266 & 0.161 & 0.213 & 0.399 & 0.489 \\
\hline $\mathrm{BA}$ & $0.000^{+++}$ & $0.000^{+++}$ & $0.000^{+++}$ & $0.000^{+++}$ & $0.000^{+++}$ & $0.000^{+++}$ & $0.000^{+++}$ & $0.000^{+++}$ & $0.000^{+++}$ \\
\hline $\mathrm{SCF}$ & 0.255 & 0.463 & 0.399 & 0.295 & 0.196 & 0.270 & 0.309 & 0.296 & 0.362 \\
\hline OFB & $0.000^{+++}$ & $0.000^{+++}$ & $0.000^{+++}$ & $0.000^{+++}$ & $0.000^{+++}$ & $0.000^{+++}$ & $0.000^{+++}$ & $0.000^{+++}$ & $0.000^{+++}$ \\
\hline $\mathrm{RE}$ & $0.073^{+}$ & $0.100^{+}$ & $0.099^{+}$ & $0.067^{+}$ & $0.046^{++}$ & $0.042^{++}$ & $0.053^{+}$ & $0.079^{+}$ & 0.103 \\
\hline SERV & 0.417 & 0.409 & 0.432 & 0.382 & 0.400 & 0.416 & 0.428 & 0.429 & 0.469 \\
\hline
\end{tabular}

Notes: This table shows the results of the Welch's tests for the debt ratio differentials as to the firms in the industries at the Tokyo Stock Exchange First Section. The sample period for the analyses is for the fiscal year from 1986 to 2012. The values in the table are $p$-values of the Welch's tests for the capital structure differentials. The null hypothesis here is that the average debt ratio of each industry equals to that of the full sample, while the alternative hypothesis is that the average debt ratio of each industry does not equal to that of the full sample. Further, $+++(---),++(--)$, and $+(-)$ denote the statistical significance of higher (lower) debt ratios than overall average at the $1 \%$ level, $5 \%$ level, and $10 \%$ level, respectively. 
Using these data, we firstly overview the equally weighted average values of corporate capital structures in various industries at the TSE First Section. Then we statistically test whether the capital structures of various industries are different from the full sample average values. Finally, we examine whether the risk connected with the level of debt ratio is rewarded with higher one-year future stock return in each industry by using pooled regressions and by excluding the industrial differential effects.

The industries at the TSE First Section analyzed in this paper are as follows; FAF: fishery, agriculture and forestry, MIN: mining, CONS: construction, FOOD: foods, TA: textiles and apparels, PP: pulp and paper, CHEM: chemicals, PHAR: pharmaceutical, OCP: oil and coal products, RP: rubber products, GCP: glass and ceramics products, IS: iron and steel, NM: nonferrous metals, MP: metal products, MACHN: machinery, EA: electric appliances, TE: transportation equipments, PI: precision instruments, OP: other products, EPG: electric power and gas, LT: land transportation, MT: marine transportation, AT: air transportation, WHTS: warehousing and harbor transportation services, IC: information and communication, WT: wholesale trade, RT: retail trade, BA: banks, SCF: securities and commodity futures, OFB: other financing business, RE: real estate, and SERV: services. Further, in this paper, the values of the capital structures, which are the focus of our analyses, are measured by total book-value debts divided by total book-value assets.

\section{An Overview of the Industrial Differentials in the Capital Structures}

First, Figure 1 exhibits the differentials of the corporate capital structures of various industries at the TSE First Section. The values in this figure are the averages of the corporate debt ratios in various industries for the period from the fiscal year of 1986 to 2012. From this figure, we graphically recognize the industrial differentials in debt ratios at the TSE First Section in Japan.

Next, describing individually, in the average values for 27 fiscal years in Figure 1, very high average debt ratios are observed in the banks (BA) and other financing business (OFB) industries. On the other hand, very low average debt ratios are observed in the pharmaceutical (PHAR) and information and communication (IC) industries. Moreover, relatively high average debt ratios are observed in the fishery, agriculture and forestry (FAF) industry, electric power and gas (EPG) industry, and air transportation (AT) industry. Contrary, relatively low average debt ratios are observed in the mining (MIN) industry.

Further, Table 1 surveys the capital structures of the industries at the TSE First Section in time-series data. We consider that it is significantly important to read the tendency from actual data first without using any technical tools. This table shows the real data trends of average debt ratios of various industries at the TSE First Section from the fiscal year of 1986 to 2012. From the data in Table 1, again, we understand that the levels of the capital structure vary in time-series. In addition, very interestingly, we recognize the continuous downward trend of overall average debt ratios at the TSE First Section. Again, we emphasize that the actual time-series data information displayed in Table 1 is significantly informative to grasp the tendency of the capital structure at the TSE First Section in Japan. The understanding for all industries as above shall be important base for our various related researches in the future as well.

\section{Statistical Tests of the Industrial Differentials in the Capital Structures}

Next, we examine whether the recognized capital structure differentials in industries are statistically significant. Our interest also lies in the relative relation between debt ratio dynamics of each industry and that of the overall average. To examine these issues, we perform the Welch's test, and the results are shown in Table 2.

In this table, ' $p$-values of the Welch's tests' denote the $p$-values for the test of average value equality. The null hypothesis here is that the average debt ratio of each industry equals to that of full sample. While the alternative hypothesis here is that the average debt ratio of each industry does not equal to that of full sample. Further, in Table $2,+++(---),++(--)$, and $+(-)$ denote the each year statistical significance of higher (lower) debt ratios than full sample average at the $1 \%$ level, $5 \%$ level, and $10 \%$ level, respectively. According to the $p$-values of the Welch's tests in Table 2, in many industries, capital structures are different from that of full sample.

More concretely, statistically significantly higher debt ratios than full sample average are observed in construction (CONS), pulp and paper (PP), electric power and gas (EPG), land transportation (LT), wholesale trade (WT), banks (BA), and other financing business (OFB). On the other hand, statistically significantly lower debt ratios are observed in foods (FOOD), pharmaceutical (PHAR), metal products (MP), electric appliances (EA), and information and communication (IC). Debt ratios of the above industries are continuously higher or lower than full sample average. In addition, from this table, we also understand that in some industries, levels of capital structure largely change as time varies. 
Table 3. Results of pooled regressions for testing the relations between capital structures and one-year future stock returns with controlling firm sizes: Evidence from the industries at the Tokyo Stock Exchange First Section for the period from the fiscal year of 1986 to 2011

\begin{tabular}{|c|c|c|c|c|c|c|c|c|c|}
\hline \multicolumn{10}{|c|}{ Results of the balanced panel regressions } \\
\hline & Constant & $p$-value & Capital structure & $p$-value & SIZE & $p$-value & $\operatorname{Adj} \cdot R^{2}$ & Obs.(CS) & Obs.(Panel) \\
\hline FAF & 39.946 & 0.455 & -35.935 & 0.613 & $-0.132 * *$ & 0.023 & 0.011 & 2 & 52 \\
\hline MIN & $18.099^{* *}$ & 0.019 & 0.393 & 0.977 & $-0.298 * *$ & 0.014 & 0.035 & 3 & 78 \\
\hline CONS & 3.189 & 0.655 & 5.209 & 0.738 & $-0.019 * *$ & 0.039 & 0.008 & 68 & 1768 \\
\hline FOOD & 4.020 & 0.226 & 0.135 & 0.985 & $-0.008 * *$ & 0.030 & 0.004 & 43 & 1118 \\
\hline TA & 0.838 & 0.872 & 10.815 & 0.299 & $-0.017 * *$ & 0.028 & 0.006 & 26 & 676 \\
\hline PP & -9.206 & 0.468 & 19.462 & 0.321 & -0.013 & 0.125 & 0.003 & 7 & 182 \\
\hline CHEM & 4.607 & 0.530 & 2.289 & 0.838 & $-0.006^{*}$ & 0.083 & 0.002 & 79 & 2054 \\
\hline PHAR & 7.371 & 0.252 & -3.419 & 0.795 & -0.001 & 0.390 & -0.003 & 19 & 494 \\
\hline $\mathrm{OCP}$ & -2.925 & 0.740 & $31.764^{*}$ & 0.088 & $-0.054 * * *$ & 0.010 & 0.027 & 5 & 130 \\
\hline $\mathrm{RP}$ & -15.130 & 0.393 & 39.482 & 0.203 & 0.001 & 0.928 & 0.018 & 8 & 208 \\
\hline GCP & 5.135 & 0.458 & 4.774 & 0.676 & $-0.011 * * *$ & 0.001 & 0.004 & 22 & 572 \\
\hline IS & -12.606 & 0.145 & $46.758^{*}$ & 0.055 & $-0.011^{* *}$ & 0.021 & 0.022 & 26 & 676 \\
\hline NM & 17.094 & 0.343 & -7.698 & 0.708 & $-0.025 * * *$ & 0.010 & 0.013 & 17 & 442 \\
\hline MP & 5.309 & 0.520 & 4.756 & 0.736 & $-0.019 * *$ & 0.038 & 0.003 & 20 & 520 \\
\hline MACHN & 7.492 & 0.249 & 4.726 & 0.569 & $-0.014 * *$ & 0.021 & 0.004 & 68 & 1768 \\
\hline EA & 9.252 & 0.210 & 1.525 & 0.850 & $-0.004 * *$ & 0.013 & 0.004 & 81 & 2106 \\
\hline TE & 0.871 & 0.908 & 18.260 & 0.236 & -0.0004 & 0.399 & 0.003 & 35 & 910 \\
\hline PI & 11.684 & 0.113 & 2.772 & 0.787 & $-0.016^{* *}$ & 0.030 & 0.003 & 12 & 312 \\
\hline $\mathrm{OP}$ & 0.151 & 0.988 & 14.257 & 0.469 & -0.003 & 0.293 & 0.001 & 21 & 546 \\
\hline EPG & 6.168 & 0.664 & -4.060 & 0.840 & $-0.003 * *$ & 0.029 & 0.021 & 14 & 364 \\
\hline LT & -1.736 & 0.798 & 13.625 & 0.192 & $-0.015^{* * *}$ & 0.002 & 0.028 & 24 & 624 \\
\hline MT & -2.363 & 0.936 & 37.840 & 0.376 & -0.038 & 0.114 & 0.010 & 9 & 234 \\
\hline AT & 9.999 & 0.859 & -16.464 & 0.820 & -0.003 & 0.401 & -0.033 & 2 & 52 \\
\hline WHTS & 14.111 & 0.229 & -6.047 & 0.718 & $-0.062 * *$ & 0.032 & 0.011 & 9 & 234 \\
\hline IC & -23.716 & 0.244 & $86.694 * *$ & 0.044 & $-0.002 * * *$ & 0.000 & 0.031 & 4 & 104 \\
\hline WT & 0.263 & 0.966 & 10.001 & 0.364 & -0.004 & 0.217 & 0.002 & 51 & 1326 \\
\hline RT & -1.614 & 0.777 & 14.471 & 0.148 & $-0.018 * * *$ & 0.000 & 0.010 & 27 & 702 \\
\hline BA & -84.976 & 0.627 & 92.780 & 0.612 & $-0.010 * * *$ & 0.005 & 0.008 & 46 & 1196 \\
\hline SCF & 31.780 & 0.386 & -19.591 & 0.715 & $-0.006^{* *}$ & 0.014 & 0.014 & 5 & 130 \\
\hline OFB & 39.151 & 0.394 & -25.942 & 0.624 & -0.014 & 0.106 & -0.003 & 5 & 130 \\
\hline $\mathrm{RE}$ & -7.619 & 0.505 & 32.242 & 0.253 & $-0.009 *$ & 0.099 & 0.011 & 11 & 286 \\
\hline SERV & 1.343 & 0.855 & 2.329 & 0.747 & -0.003 & 0.615 & -0.006 & 11 & 286 \\
\hline
\end{tabular}

Notes: This table shows the results of balanced panel regressions as to the firms in various industries at the TSE First Section. The sample period is from the fiscal year of 1986 to 2011 and the dependent variable is the one-year future returns. 'Capital structure' denotes the corporate debt ratios, which are measured by total book-value debts divided by total book-value assets. Adj. $R^{2}$ denotes the adjusted $R$-squared values, Obs. (Panel) is the pooled data number, and Obs. (CS) is the number of cross-sectional data in each year. In estimation, we used the White cross-section standard errors and covariance. ***, **, and * denote the statistical significance at the $1 \%, 5 \%$, and $10 \%$ level, respectively. 
Table 4. Results of balanced pooled regressions for testing the relations between capital structures and one-year future stock returns: Evidence from the industries at the Tokyo Stock Exchange First Section for the period from the fiscal year of 1986 to 2011

\begin{tabular}{|c|c|c|c|c|c|c|c|}
\hline \multicolumn{8}{|c|}{ Results of the balanced panel regressions } \\
\hline & Constant & $p$-value & Capital structure & $p$-value & Adj. $R^{2}$ & Obs.(CS) & Obs.(Panel) \\
\hline FAF & 8.254 & 0.882 & -8.668 & 0.907 & -0.020 & 2 & 52 \\
\hline MIN & -0.160 & 0.982 & 11.431 & 0.452 & -0.008 & 3 & 78 \\
\hline CONS & 0.921 & 0.903 & 4.878 & 0.753 & -0.0002 & 68 & 1768 \\
\hline FOOD & 2.396 & 0.472 & 0.686 & 0.924 & -0.001 & 43 & 1118 \\
\hline TA & -1.986 & 0.685 & 12.568 & 0.236 & 0.002 & 26 & 676 \\
\hline PP & -14.202 & 0.252 & 23.906 & 0.226 & 0.000 & 7 & 182 \\
\hline CHEM & 2.473 & 0.719 & 4.345 & 0.694 & 0.000 & 79 & 2054 \\
\hline PHAR & 5.838 & 0.374 & -1.132 & 0.935 & -0.002 & 19 & 494 \\
\hline $\mathrm{OCP}$ & 5.783 & 0.485 & -1.305 & 0.909 & -0.008 & 5 & 130 \\
\hline $\mathrm{RP}$ & -14.664 & 0.300 & 38.869 & 0.147 & 0.023 & 8 & 208 \\
\hline GCP & 0.607 & 0.930 & 9.281 & 0.431 & -0.001 & 22 & 572 \\
\hline IS & -11.917 & 0.168 & $41.503 *$ & 0.075 & 0.015 & 26 & 676 \\
\hline NM & 8.841 & 0.593 & -3.446 & 0.867 & -0.002 & 17 & 442 \\
\hline MP & 0.754 & 0.913 & 10.249 & 0.435 & -0.0004 & 20 & 520 \\
\hline MACHN & 5.305 & 0.411 & 5.740 & 0.502 & 0.000 & 68 & 1768 \\
\hline EA & 7.350 & 0.324 & 2.152 & 0.794 & -0.0004 & 81 & 2106 \\
\hline $\mathrm{TE}$ & -0.606 & 0.933 & 20.246 & 0.191 & 0.004 & 35 & 910 \\
\hline PI & 8.513 & 0.205 & 2.462 & 0.803 & -0.003 & 12 & 312 \\
\hline $\mathrm{OP}$ & -3.766 & 0.649 & 20.087 & 0.276 & 0.002 & 21 & 546 \\
\hline EPG & 11.152 & 0.366 & -14.568 & 0.365 & 0.001 & 14 & 364 \\
\hline LT & -1.948 & 0.775 & 7.469 & 0.477 & 0.001 & 24 & 624 \\
\hline MT & -13.283 & 0.645 & 43.058 & 0.331 & 0.001 & 9 & 234 \\
\hline AT & 9.916 & 0.855 & -18.332 & 0.792 & -0.018 & 2 & 52 \\
\hline WHTS & -0.657 & 0.942 & 10.597 & 0.516 & -0.002 & 9 & 234 \\
\hline IC & 2.362 & 0.901 & 5.850 & 0.865 & -0.010 & 4 & 104 \\
\hline WT & 0.927 & 0.877 & 7.967 & 0.429 & 0.001 & 51 & 1326 \\
\hline RT & -3.437 & 0.554 & 13.199 & 0.193 & 0.002 & 27 & 702 \\
\hline BA & -104.946 & 0.543 & 111.575 & 0.537 & 0.002 & 46 & 1196 \\
\hline SCF & 28.800 & 0.430 & -25.930 & 0.627 & -0.002 & 5 & 130 \\
\hline OFB & 24.731 & 0.585 & -14.824 & 0.779 & -0.008 & 5 & 130 \\
\hline $\mathrm{RE}$ & -7.279 & 0.515 & 25.873 & 0.301 & 0.003 & 11 & 286 \\
\hline SERV & 0.523 & 0.938 & 3.039 & 0.680 & -0.003 & 11 & 286 \\
\hline
\end{tabular}

Notes: This table shows the results of balanced panel regressions as to the firms in various industries at the TSE First Section. The sample period is from the fiscal year of 1986 to 2011 and the dependent variable is the one-year future returns. 'Capital structure' denotes the corporate debt ratios, which are measured by total book-value debts divided by total book-value assets. Adj. $R^{2}$ denotes the adjusted $R$-squared values, Obs. (Panel) is the pooled data number, and Obs. (CS) is the number of cross-sectional data in each year. In estimation, we used the White cross-section standard errors and covariance. ***, **, and * denote the statistical significance at the $1 \%, 5 \%$, and $10 \%$ level, respectively. 


\section{Testing the Relations between Capital Structure and Stock Return by Excluding the Industrial Differential Effects}

This section examines the linkage between debt ratio and one-year future stock return within each industry. Are debt ratios rewarded with positive returns as the general suggestion of the second proposition by Modigliani and Miller (1958)? In addition, we note that our investigations so far imply the importance of excluding the industrial differential effects on corporate leverage to understand the real relation between capital structure and stock return. In order to clarify the actual relation between them, we use two kinds of panel regression models. First is the following model (1) with a control variable of firm size, SIZE:

$$
R E T_{i, t+1}=l_{i}+\xi_{i} C S_{i, t}+\pi_{i} S I Z E_{i, t}+\tau_{i, t+1} .
$$

In model (1), $R E T_{i, t+1}$ denotes the one-year future stock return of firm $i$ in a certain industry in year $t+1$. Further, $C S_{i, t}$ denotes the debt ratio (measured by total book-value debts divided by total book-value assets) of firm $i$ in a certain industry in year $t$. Moreover, $S I Z E_{i, t}$ denotes the firm size (measured by market capitalization at the end of the fiscal year) of firm $i$ in a certain industry in year $t$. Our model (1) is very simple; however, simple models generally derive variable relations more soundly. We also emphasize that not using such variables as dummy variables but using the same industry samples shall be more effective to exclude the industrial effects on capital structure.

Estimation results of our pooled regression model (1) are presented in Table 3. As seen in this table, only in the oil and coal products (OCP) industry, iron and steel (IS) industry, and information and communication (IC) industry, corporate capital structures are positively related with their future stock returns. Contrary to this, SIZE is negatively related with the future stock returns in many industries, and this shows the well-known size effects.

We point out here that strong size effects seen in Table 3 might hide the true relation between capital structure and stock return. Therefore, in order to scrutinize the one-to-one relationship between capital structure and the one-year future stock return within each industry, we exploit the following model (2). This model includes no control variable as follows:

$$
R E T_{i, t+1}=v_{i}+\zeta_{i} C S_{i, t}+\kappa_{i, t+1} .
$$

The notations of variables are the same as those in model (1).

Again, we emphasize that our pooled regression model (2) is very simple; however, simple models generally derive the relations between variables more robustly. The results are displayed in Table 4. As Table 4 shows, when we exclude the size effects observed in Table 3, we find almost no positive relation between corporate capital structures and future stock returns. That is, our investigations reveal that, when we exclude the effects of industrial differentials of corporate leverage, we find almost no positive relation between corporate debt ratios and future stock returns at the TSE First Section in Japan.

\section{Summary and Conclusions}

This paper presented an overview of the differentials in capital structures in various industries in Japan. More specifically, we firstly examined whether the capital structure of each industry at the TSE First Section is statistically significantly different. After that, we investigated whether the financial risk associated with the corporate debt ratio is rewarded with higher stock return within industries. The findings from our studies are summarized as follows. 1) First, we found that the capital structure of each industry at the TSE First Section was statistically significantly much different. 2) Second, we also revealed that the financial risk connected with the corporate leverage was not rewarded with future return when we exclude not only the effects of industrial differentials but also the size effects.

As for the implications from our study, in the practical investments, to pursue the higher return, as far as in Japan, it may be useless to look at the corporate capital structure because the higher financial risks due to the higher debt ratios are not rewarded with higher return. While as for the academic implication, our evidence suggests that the actual relationship between the debt ratio and stock return in Japan seem to be close to the suggestion of the first proposition of Modigiliani and Miller (1958). They documented that corporate capital structures are irrelevant to the firm values. Although the capital structure is academically important topic, however, from the practical viewpoint, looking for other firm characteristics rewarded with higher returns may have priority and this shall be one of our future works.

\section{Acknowledgements}

The author thanks the Japan Society for the Promotion of Science for their generous financial assistance for this research. Further, the author greatly thanks the repeated kind invitation from the journal to write to this journal. I 
also thank the Editor and the anonymous referees for their comments to this paper. Finally, I greatly appreciate the Editor for making the quick decision to my paper.

\section{References}

Eisdorfer, A., Giaccotto, C., \& White, R. (2013). Capital structure, executive compensation, and investment efficiency. Journal of Banking \& Finance, 37, 549-562. http://dx.doi.org/10.1016/j.jbankfin.2012.09.011

Feld, L. P., Heckemeyer, J. H., \& Overesch, M. (2013). Capital structure choice and company taxation: A meta-study. Journal of Banking \& Finance, 37, 2850-2866. http://dx.doi.org/10.1016/j.jbankfin.2013.03.017

Fier, S. G., McCullough, K. A., \& Carson, J. M. (2013). Internal capital markets and the partial adjustment of leverage. Journal of Banking \& Finance, 37, 1029-1039. http://dx.doi.org/10.1016/j.jbankfin.2012.11.003

Kayo, E. K., \& Kimura, H. (2011). Hierarchical determinants of capital structure. Journal of Banking \& Finance, 35, 358-371. http://dx.doi.org/10.1016/j.jbankfin.2010.08.015

Margaritis, D., \& Psillaki, M. (2010). Capital structure, equity ownership and firm performance. Journal of Banking \& Finance, 34, 621-632. http://dx.doi.org/10.1016/j.jbankfin.2009.08.023

Modigliani, F., \& Miller, M. H. (1958). The cost of capital, corporation finance and the theory of investment. The American Economic Review, 48, 261-297.

Schmid, T. (2013). Control considerations, creditor monitoring, and the capital structure of family firms. Journal of Banking \& Finance, 37, 257-272. http://dx.doi.org/10.1016/j.jbankfin.2012.08.026

Wang, H. (2011). Managerial entrenchment, equity payout and capital structure. Journal of Banking \& Finance, 35, 36-50. http://dx.doi.org/10.1016/j.jbankfin.2010.07.018

Wu, X., \& Yeung, C. K. A. (2012). Firm growth type and capital structure persistence. Journal of Banking \& Finance, 36, 3427-3443. http://dx.doi.org/10.1016/j.jbankfin.2012.08.008

\section{Copyrights}

Copyright for this article is retained by the author(s), with first publication rights granted to the journal.

This is an open-access article distributed under the terms and conditions of the Creative Commons Attribution license (http://creativecommons.org/licenses/by/3.0/). 